\title{
$5 \alpha$-Reductase Inhibition Prevents the Luteal Phase Increase in Plasma Allopregnanolone Levels and Mitigates Symptoms in Women with Premenstrual Dysphoric Disorder
}

\author{
Pedro E Martinez', David R Rubinow ${ }^{2}$, Lynnette K Nieman ${ }^{3}$, Deloris E Koziol ${ }^{4}$, A Leslie Morrow ${ }^{2}$, \\ Crystal E Schiller', Dahima Cintron ${ }^{5}$, Karla D Thompson', Khursheed K Khine' and Peter J Schmidt, \\ 'Behavioral Endocrinology Branch, NIMH, Bethesda, MD, USA; '2Department of Psychiatry, University of North Carolina, Chapel Hill, NC, USA; \\ ${ }^{3}$ Intramural Research Program on Reproductive and Adult Endocrinology, The Eunice Kennedy Shriver National Institute of Child Health and \\ Human Development, NIH, DHSS, Bethesda, MD, USA; ${ }^{4}$ Biostatistics \& Clinical Epidemiology Service, Clinical Center, National Institutes of Health, \\ Bethesda, MD, USA; ${ }^{5}$ University of Puerto Rico School of Medicine, San Juan Puerto Rico, USA
}

\begin{abstract}
Changes in neurosteroid levels during the luteal phase of the menstrual cycle may precipitate affective symptoms. To test this hypothesis, we stabilized neurosteroid levels by administering the $5 \alpha$-reductase inhibitor dutasteride to block conversion of progesterone to its neurosteroid metabolite allopregnanolone in women with premenstrual dysphoric disorder (PMDD) and in asymptomatic control women. Sixteen women with prospectively confirmed PMDD and 16 control women participated in one of two separate randomized, doubleblind, placebo-controlled, cross-over trials, each lasting three menstrual cycles. After one menstrual cycle of single-blind placebo, participants were randomized to receive, for the next two menstrual cycles, either double-blind placebo or dutasteride (low-dose $0.5 \mathrm{mg} /$ day in the first eight PMDD and eight control women or high-dose $2.5 \mathrm{mg} /$ day in the second group of women). All women completed the daily rating form (DRF) and were evaluated in clinic during the follicular and luteal phases of each menstrual cycle. Main outcome measures were the DRF symptoms of irritability, sadness, and anxiety. Analyses were performed with SAS PROC MIXED. In the low-dose group, no significant effect of dutasteride on PMDD symptoms was observed compared with placebo (ie, symptom cyclicity maintained), and plasma allopregnanolone levels increased in women with PMDD from follicular to the luteal phases, suggesting the absence of effect of the lowdose dutasteride on $5 \alpha$-reductase. In contrast, the high-dose group experienced a statistically significant reduction in several core PMDD symptoms (ie, irritability, sadness, anxiety, food cravings, and bloating) on dutasteride compared with placebo. Dutasteride had no effect on mood in controls. Stabilization of allopregnanolone levels from the follicular to the luteal phase of the menstrual cycle by blocking the conversion of progesterone to its $5 \alpha$-reduced neurosteroid metabolite mitigates symptoms in PMDD. These data provide preliminary support for the pathophysiologic relevance of neurosteroids in this condition.

Neuropsychopharmacology (2016) 4I, I093-I I02; doi: I0.I038/npp.20 I5.246; published online 9 September 2015
\end{abstract}

\section{INTRODUCTION}

Premenstrual dysphoric disorder (PMDD) is characterized by distressing mood and behavioral symptoms that occur during the luteal phase of the normal menstrual cycle and disappear within a few days after menses begin (American Psychiatric Association, 1994; American Psychiatric Association, 2013). No abnormalities of ovarian hormone levels consistently identify women with PMDD (Rubinow and Schmidt, 2006). Nonetheless, GnRH agonist-induced ovarian suppression in women with PMDD eliminates symptoms, which can then be

*Correspondence: Dr PJ Schmidt, Behavioral Endocrinology Branch, NIMH, Bldg. IOCRC, Room 25330, 10 Center Drive MSC I277, Bethesda, MD 20892-1277, USA, Tel: + I 301496 6I20, Fax: + I 30 I 480 4597, E-mail: peterschmidt@mail.nih.gov

Received 24 April 2015; revised 7 July 2015; accepted I8 July 2015; accepted article preview online 14 August 2015 precipitated by the administration of physiologic doses of ovarian steroids (Schmidt et al, 1998). Reproductive steroids, therefore, appear to have a role in PMDD.

The ring-A-reduced neurosteroid metabolite of progesterone, allopregnanolone (ie, $3 \alpha$-hydroxy-5 $\alpha$-pregnan-20one), potentially mediates the symptom precipitating effects of progesterone in PMDD. Allopregnanolone regulates central GABA-A receptor function and hence CNS excitation (Majewska et al, 1986; Paul and Purdy, 1992; Maguire et al, 2005; Shen et al, 2007). In one study, higher plasma levels of allopregnanolone was associated with less severe PMDD symptoms (Wang et al, 1996); however, abnormal plasma allopregnanolone levels have not been consistently observed in PMDD (Schmidt et al, 1994; Wang et al, 1996; Rapkin et al, 1997; Monteleone et al, 2000; Epperson et al, 2002; Lombardi et al, 2004; Schiller et al, 2014). Preclinical studies indicate that the impact of neurosteroids on the GABA receptor is mediated by changes in their levels rather than 
their absolute concentration. Both increases and decreases in progesterone metabolite neurosteroids induce changes in the alpha 4 subunit conformation of the $\mathrm{GABA}_{\mathrm{A}} \mathrm{R}$ sufficient to produce anxiety-like behaviors (Gulinello et al, 2001; Gulinello and Smith, 2003; Shen et al, 2005). Thus, it is possible that effects of progesterone to precipitate PMDD symptoms (Schmidt et al, 1998) could be mediated by changes in allopregnanolone and/or an abnormal conversion of progesterone to its neurosteroid metabolites.

In this study, we attempted to stabilize allopregnanolone levels in women with PMDD by blocking its formation from progesterone with the $5 \alpha$-reductase (type 1 and 2) competitive inhibitor dutasteride. We hypothesized that this would abrogate the luteal phase increase in allopregnanolone levels resulting from increased progesterone secretion (Schmidt et al, 1994) and thus prevent PMDD symptoms. We also examined the effects of dutasteride in control women who did not experience menstrual cycle-related mood or behavioral symptoms.

\section{MATERIALS AND METHODS}

\section{Participant Selection}

We studied 16 women with PMDD and 16 women without the syndrome (hereafter referred to as control women), aged 30-50 years. All reported regular menstrual cycles (range, 21-35 days), good medical health, and were medication-free. All women had normal physical findings, including gynecologic and breast examination, normal laboratory test results and a negative pregnancy test upon study entry. All agreed to use barrier contraception throughout the study. None of the women with PMDD had any Axis I psychiatric illness currently or within the previous 2 years as determined by the Structured Clinical Interview for the Diagnostic and Statistical Manual of Mental Disorders (fourth edition) (DSM-IV) (First et al, 2002). No control women had any premenstrual mood symptoms using the same daily rating scales described below during a 2-month baseline period, and none had any current or past Axis I psychiatric illness.

Before randomization, women with PMDD prospectively confirmed the timing and severity of their mood-related symptoms by rating themselves daily for 3 months using a four-item visual-analog scale (irritability, sadness, anxiety, and mood swings) (Rubinow et al, 1984; Schmidt et al, 1998). All women met study criteria for PMDD, which are based on requirements outlined in the American Psychiatric Association's Diagnostic and Statistical Manual of Mental Disorders, fourth edition (DSM-IV) (American Psychiatric Association, 1994). The mean score of at least one of these self-rated negative mood symptoms was at least $30 \%$ higher (relative to the range of the scale used by each woman) in the week before menstruation compared with the week after the cessation of menstruation in at least two of the three cycles assessed. Functional impairment was assessed through selfreports of distress and functional impairment on the daily rating form (DRF) (Endicott et al, 1986). The DRF criteria for functional impairment were as follows: a DRF score of 2 (minimal) or higher on one of 4 questions related to functional impairment (ie, stayed at home or avoided social activities, had conflicts or problems with people, symptoms interfered with relationships at work or home, or symptoms interfered with work productivity) in at least 3 days out of 7 days pre-menses. Finally, DRF ratings and the results of both a semi-structured interview and a self-report questionnaire (ie, the Menstrual Screening Questionnaire and the Menstrual Assessment Form, respectively) were employed to confirm that all women met the required number of symptoms specified in the DSM criteria for PMDD. Additionally, women with significant negative mood symptoms occurring during the follicular phase of the menstrual cycle (on the DRF) were excluded. Thus, in this study, the diagnostic criteria for PMDD were augmented by the severity criterion of a $30 \%$ increase in mean negative mood during the week before menses compared with the week after menses, a more stringent criterion than that of DSM-4 or 5 (American Psychiatric Association, 1994; American Psychiatric Association, 2013). The global assessment of functioning scale (GAF) score (Hilsenroth et al, 2000) was

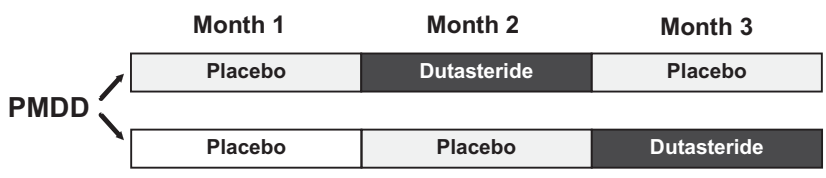

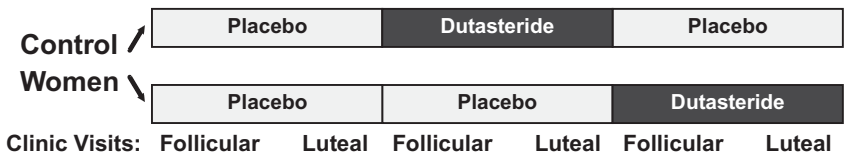

Figure I Study schematic - effects of the $5 \alpha$-reductase inhibitor dutasteride on symptoms of premenstrual dysphoric disorder. Women were either self-referred to our clinic in response to advertisements in the local newspapers or were referred by their personal physicians. We studied women with premenstrual dysphoric disorder (PMDD) and women who did not have the syndrome (control women). Following I month of lead-in single-blind placebo, all women received dutasteride and placebo in a randomized, double-blind, cross-over study for one menstrual cycle each beginning on day I of menses. Two sets of random number lists were employed for the randomization within each treatment trial (low and high dose), one for PMDD participants and one for control women, and lists were blocked into four participants each. Dutasteride was given at two different doses in two separate trials: the low dose at $0.5 \mathrm{mg}$ and the high dose at $2.5 \mathrm{mg}$. Each evening during the 2-3 months of baseline before receiving treatment and during the three menstrual cycles on treatment, all women completed the daily rating form (DRF) (Endicott et al, 1986). The DRF is a six point Likert-type scale. Scores of $1=$ symptoms absent; $6=$ symptoms present in the extreme. All women were instructed that the ratings should represent a composite rating for the previous $12 \mathrm{~h}$. The ratings on the DRF assessed the severity of common symptoms of PMDD including irritability, sadness, anxiety, mood swings, food cravings, bloating, decreased sexual interest, and breast pain. Our primary outcome measure of interest was DRF scores for the core PMDD symptoms of irritability, sadness, and anxiety (American Psychiatric Association, 20/3). DRF symptom ratings were employed both to confirm that women with PMDD met Diagnostic and Statistical Manual of Mental Disorders (fourth edition) criteria for PMDD and as the main study outcome measures. During the three menstrual cycles of treatment, clinic visits were scheduled during follicular days 4-10 and luteal phase days 6-10 post-LH surge as determined by a home ovulation kit (Clearblue Easy Fertility Monitor Inverness Medical Innovations, Inc., Waltham, Massachusetts) during which blood samples were obtained and vital signs and side effects were monitored. All participants were followed for 6 months after completing the medication portion of the protocol (while remaining on barrier contraception) to monitor hepatic function and to insure that serum pregnancy tests remained negative. 
performed in each woman with PMDD to measure functional impairment related to PMDD symptoms; however, global assessment of functioning scale scores were not employed for establishing the diagnosis of PMDD.

All women received payment for participation according to the NIH Healthy Volunteer Office guidelines. The study protocol was reviewed and approved by the NIMH Institutional Review Board, and all women provided written consent to study participation.

\section{Study Design}

Because of the long elimination half-life (up to 5 weeks) (Bramson et al, 1997; GlaxoSmithKline Research Triangle Park NC, 2014) of dutasteride, all women first received one menstrual cycle single-blind placebo and then were randomized to double-blind placebo or dutasteride (at one of two doses) in a cross-over design (Figure 1). For women who received dutasteride first (ie, study month two), we analyzed the first single-blind placebo month results to avoid residual dutasteride confounding the effects of placebo. In the first trial, dutasteride (AVODART (GlaxoSmithKline, Research Triangle Park, NC, 2014)) was administered at a dose of $0.5 \mathrm{mg}$ daily (low dose) and in the second trial at a dose of
$2.5 \mathrm{mg}$ daily (high dose) for one complete menstrual cycle beginning on day one of menses and stopping on day one of menses in the next menstrual cycle. Dutasteride levels are increased within the CNS after oral administration in rodents, (GlaxoSmithKline Research Triangle Park NC, 2005) but no studies have measured the penetrance of the drug into the human CNS. As a result, this sequential twodose strategy was used.

\section{Symptom Rating Scales}

Each evening during the 2-3 months of baseline and during the three menstrual cycles on treatment, all women completed the DRF (Endicott et al, 1986). The DRF is a six point Likert-type scale. Scores of $1=$ symptoms absent; $6=$ symptoms present in the extreme. All women were instructed that the ratings should represent a composite score for the previous $12 \mathrm{~h}$. The ratings on the DRF assessed the severity of common symptoms of PMDD including irritability, sadness, anxiety, mood swings, food cravings, bloating, decreased sexual interest, and breast pain. Our primary outcome measure of interest was DRF scores for the core PMDD symptoms of irritability, sadness, and anxiety (American Psychiatric Association, 2013). DRF symptom

Table I Demographic and Clinical Characteristics of Women with Premenstrual Dysphoric Disorder and Control Women

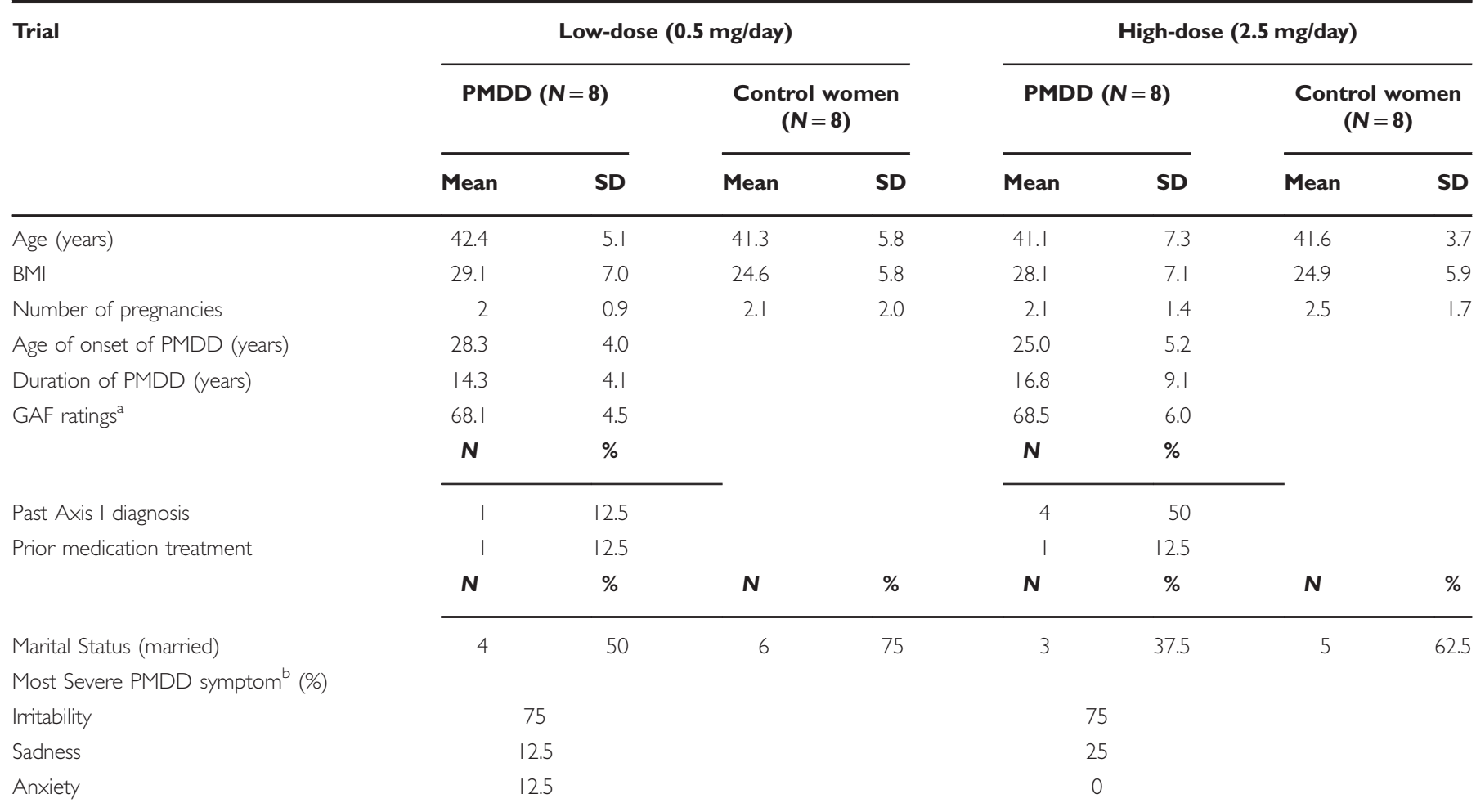

Abbreviations: BMI, body mass index; PMDD, premenstrual dysphoric disorder.

${ }^{a}$ Global assessment of functioning scale (GAF) score measuring overall functional impairment related to PMDD (scores in the range of $8 \mathrm{I}-100=$ high function; $5 \mathrm{I}-$ $70=$ mild to moderate symptoms; I-50 = low function/ suicidality). Baseline PMDD GAF scores were not significantly different between those women receiving the low $(0.5 \mathrm{mg})$ and high $(2.5 \mathrm{mg})$ daily doses of dutasteride $(p=\mathrm{NS})$.

blentified by self-report during initial screening interview in women with PMDD.

Women's ages ranged from 30 to 50 years: (mean $(S D)$ ) women with PMDD $=42.4(5.1)$ years and control women $=38.3(2.3)$ years. Women with PMDD and control women did not differ in any demographic characteristics including age (low-dose $p>0.05$; high-dose $p>0.05$ ), parity (low-dose $p>0.05$; high-dose $p>0.05$ ), and BMI ( $p>0.05)$. Women treated for PMDD symptoms in the past (one woman received fluoxetine (high-dose group) and one received buspirone (low-dose group)). 
ratings were employed both to confirm that women with PMDD met DSM-IV criteria for PMDD and as the main study outcome measures. During the three menstrual cycles of treatment, clinic visits were scheduled during follicular days $4-10$ and luteal phase days $6-10$ post-LH surge (as determined by a home ovulation kit (Clearblue Easy Fertility Monitor Inverness Medical Innovations, Inc., Waltham, MA)), during which blood samples were obtained and vital signs and side effects were monitored.

\section{Hormone Assays}

Plasma levels of neurosteroids (ie, pregnenolone, allopregnanolone, pregnanolone (ie, $3 \alpha$-hydroxy-5 $\beta$-pregnan20 -one), $3 \alpha, 5 \alpha$-androsterone, and $3 \alpha, 5 \beta$-androsterone) were analyzed by gas chromatography with mass spectrometry (GC-MS) at the University of North Carolina at Chapel Hill as described previously (Porcu et al, 2009). Plasma progesterone levels were assayed at the NIH Clinical Center Department of Laboratory Medicine by LC-MS/MS, and luteal plasma levels $<2 \mathrm{ng} / \mathrm{ml}$ were considered to be consistent with either anovulation or a clinic visit too late or early in the luteal phase and, therefore, were not included in comparisons of plasma neurosteroid levels (see below). Plasma estradiol levels were measured by LC-MS/MS at NMS Laboratories (Willow Grove, PA).

\section{Statistical Analysis}

Symptom response was assessed by the 7-day average DRF scores after the end of menses (ie, week 2 of the menstrual cycle, postmenstrual) and for the week before menses begins (ie, week 4 of the menstrual cycle, premenstrual). Thus, our data involved repeated measures (follicular (week 2) and late luteal (week 4)) on the same woman during two treatments (placebo and dutasteride). Repeated measures analyses were done with SAS Version 9.2 software (SAS Institute, Inc., Cary, NC), using PROC MIXED (for mixed models). Separately, for each symptom rating outcome, the predictor variables of interest, treatment (placebo or dutasteride) and order of treatment (dutasteride or placebo first) were modeled as fixed effects. We used the Kenward-Roger method for computing the degrees of freedom for tests of fixed effects.

For each of the eight symptoms we also analyzed the effect of the two treatment assignments. First, to examine whether the difference in symptom severity between week 4 and week 2 changed during dutasteride treatment compared with placebo, we performed repeated measures analyses with adjustment (in the women with PMDD) for the severity of the late luteal phase symptom severity at baseline before randomization. Second, to further determine if the effects on week 4 - week 2 difference scores reflected reductions in week 4 (ie, premenstrual) symptom severity, we examined the effect of treatment on week 4 scores only. Plasma neurosteroid levels (both raw and log-transformed values) during week 4- week 2 also were compared across drug conditions. For these analyses the value of the estimator ('least square means' in SAS terminology) and associated standard error of the estimator and $p$-values are reported.

Table 2 Weekly DRF Symptom Severity Ratings during High-dose Dutasteride in Women with Premenstrual Dysphoric Disorder

Symptom Average weekly symptom severity ratings by menstrual cycle phase (arithmetic mean (SD) ${ }^{a}$
Follicular - luteal difference in average weekly symptom severity LSE (SE)

\begin{tabular}{|c|c|c|c|c|c|c|c|c|c|c|c|c|c|c|c|c|}
\hline & \multicolumn{4}{|c|}{ Baseline } & \multicolumn{4}{|c|}{ Placebo } & \multicolumn{4}{|c|}{ Dutasteride (2.5 mg/day) } & \multicolumn{2}{|c|}{ Placebo } & \multicolumn{2}{|c|}{ Dutasteride } \\
\hline & \multicolumn{2}{|c|}{ Follicular } & \multicolumn{2}{|c|}{ Luteal } & \multicolumn{2}{|c|}{ Follicular } & \multicolumn{2}{|c|}{ Luteal } & \multicolumn{2}{|c|}{ Follicular } & \multicolumn{2}{|c|}{ Luteal } & LSE & SE & LSE & SE \\
\hline Irritability & 1.2 & 0.3 & 3.0 & 0.5 & 1.3 & 0.4 & 2.8 & 1.0 & 1.2 & 0.4 & 1.7 & 0.6 & 1.5 & 0.3 & 0.5 & $0.3^{b}$ \\
\hline Sadness & 1.2 & 0.1 & 2.4 & 1.0 & 1.1 & 0.2 & 2.2 & 1.0 & 1.0 & 0.0 & 1.4 & 0.4 & 1.5 & 0.3 & 0.5 & $0.3^{b}$ \\
\hline Mood swings & I.1 & 0.2 & 2.4 & 0.8 & I.1 & 0.3 & 2.1 & 1.0 & 1.0 & 0.0 & 1.5 & 0.5 & 1.0 & 0.3 & 0.5 & 0.3 \\
\hline Food cravings & 1.2 & 0.3 & 2.3 & 1.1 & 1.0 & 0.1 & 2.2 & 0.9 & 1.1 & 0.3 & 1.1 & 0.1 & 1.2 & 0.3 & 0.3 & $0.3^{b}$ \\
\hline Bloating & 1.1 & 1.0 & 2.1 & 0.5 & 1.0 & 0.1 & 2.8 & 1.0 & 1.0 & 0.0 & 1.3 & 0.4 & 1.6 & 0.3 & 0.5 & $0.3^{b}$ \\
\hline Breast pain & 1.2 & 0.3 & 1.9 & 0.8 & 1.0 & 0.0 & 2.1 & 1.0 & 1.0 & 0.0 & 1.3 & 0.5 & 1.3 & 0.4 & 0.6 & 0.4 \\
\hline Decreased libido & 1.2 & 0.2 & 2.1 & 0.8 & 1.0 & 0.1 & 2.6 & 1.0 & 1.0 & 0.0 & 1.3 & 0.4 & 1.0 & 0.3 & 0.3 & 0.3 \\
\hline
\end{tabular}

${ }^{a}$ Scores based on the daily rating form (DRF). The DRF is a six point Likert-type scale. Scores of I = symptoms absent; $6=$ symptoms present in extreme. All women completed the DRF each evening (the women were instructed that the ratings should represent a composite rating for the previous $12 \mathrm{~h}$ ). ${ }^{b}$ Week 2 versus week 4 differences between dutasteride and placebo $p<0.05$. Significant reductions in the difference between week 4 (premenstrual, luteal) to week 2 (postmenstrual, follicular) symptom severity scores were observed in women with PMDD during dutasteride compared with placebo treatments in the following DRF symptom scores: irritability $(p=0.04)$, anxiety $(p=0.03)$, sadness $(p=0.04)$, bloating $(p=0.03)$, and food cravings $(p=0.02)$. These differences reflected significantly decreased symptom scores in the women with PMDD during week 4 during dutasteride treatment compared with placebo (irritability $(p=0.03)$, anxiety $(p=0.06)$, sadness $(p=0.03)$, bloating $(p=0.03)$, and food cravings $(p=0.0 \mathrm{I})$ ). Non-significant improvements during dutasteride treatment compared with placebo also were observed for several symptoms including mood swings $(p=0.3)$, breast pain $(p=0.1)$, and decreased sexual interest $(p=0.1)$. 
Clinical characteristics in women with PMDD and control women were compared with Fisher's exact test for categorical variables and Student's $t$-tests for continuous variables (Table 1). Plasma progesterone levels during week 4 of each treatment cycle were compared with Student's $t$-test. A twosided $p<0.05$ was considered statistically significant.

We attempted to obtain blood samples once in both the follicular and luteal phase to confirm the expected effect of dutasteride on the luteal phase increase in allopregnanolone. Blood samples were not obtained in every woman at a time that allowed us to confirm the luteal phase increase in progesterone and its metabolites. In two of the eight women with PMDD who received high-dose dutasteride, luteal plasma progesterone levels were $<2 \mathrm{ng} / \mathrm{ml}$ during both placebo and dutasteride treatments, despite the presence of PMDD symptoms in both women during placebo and in one woman during dutasteride (albeit in partial remission). Since there was no detectable luteal phase increase in plasma progesterone, these samples were not used to test the hypothesis that highdose dutasteride prevented a luteal phase increase in allopregnanolone. However, since PMDD symptoms occurred in both of these women despite 'anovulatory' progesterone levels, we included their DRF ratings in the analyses of PMDD symptom scores. We presume that the clinic visit (and blood drawing) in these women was scheduled either too early or too late in the luteal phase or that symptoms occurred during an anovulatory cycle.

\section{RESULTS}

\section{Participant Characteristics}

Women with PMDD and control women did not differ significantly in demographic characteristics including age, parity, or body mass index. Four women with PMDD met criteria for a past depressive episode, one of whom reported a past postpartum depression (Table 1). As per our exclusion criteria, however, none of the women had current major depressive disorder (or other current Axis I disorders). Eight women with PMDD and eight control women participated in each of the two trials.

\section{Treatment Trial}

\section{Symptom ratings}

Low-dose dutasteride. No significant differences in PMDD core symptom severity measures were observed in women with PMDD during low-dose dutasteride compared with placebo (irritability $(p=0.2)$, sadness $(p=0.2)$, and anxiety $(p=0.6))$. All women with PMDD on low-dose dutasteride continued to meet diagnostic criteria and reported premenstrual mood and physical symptoms similar to baseline. Control women remained asymptomatic on both dutasteride and placebo ( $p>0.05$ all comparisons). Plasma allopregnanolone levels increased during low-dose dutasteride in women with PMDD from follicular to the luteal phases (ie, $70.7 \mathrm{pg} / \mathrm{ml}$ and $85.8 \mathrm{pg} / \mathrm{ml}$, respectively), suggesting the absence of effect of the low-dose dutasteride on $5 \alpha$-reductase. Thus, the second study with high-dose dutasteride was performed.
High-dose dutasteride. Significant reductions in the week 4 to week 2 difference in symptom severity scores were observed in women with PMDD during high-dose dutasteride compared with placebo treatments in the following DRF symptom scores: irritability $(p=0.04)$, anxiety $(p=0.03)$, sadness $(p=0.04)$, bloating $(p=0.03)$, and food cravings $(p=0.02)$ (Table 2; Figure 2). These differences reflected significantly decreased week 4 (late luteal) symptom scores in the women with PMDD during high-dose dutasteride treatment compared with placebo (irritability $(p=0.03)$, anxiety $(p=0.06)$, sadness $(p=0.03)$, bloating $(p=0.03)$, and food cravings $(p=0.01)$ ). Non-significant improvements during dutasteride treatment compared with placebo also were observed for several symptoms including mood swings $(p=0.3)$, breast pain $(p=0.1)$, and decreased sexual interest $(p=0.1)$. Six of eight women no longer met criteria for
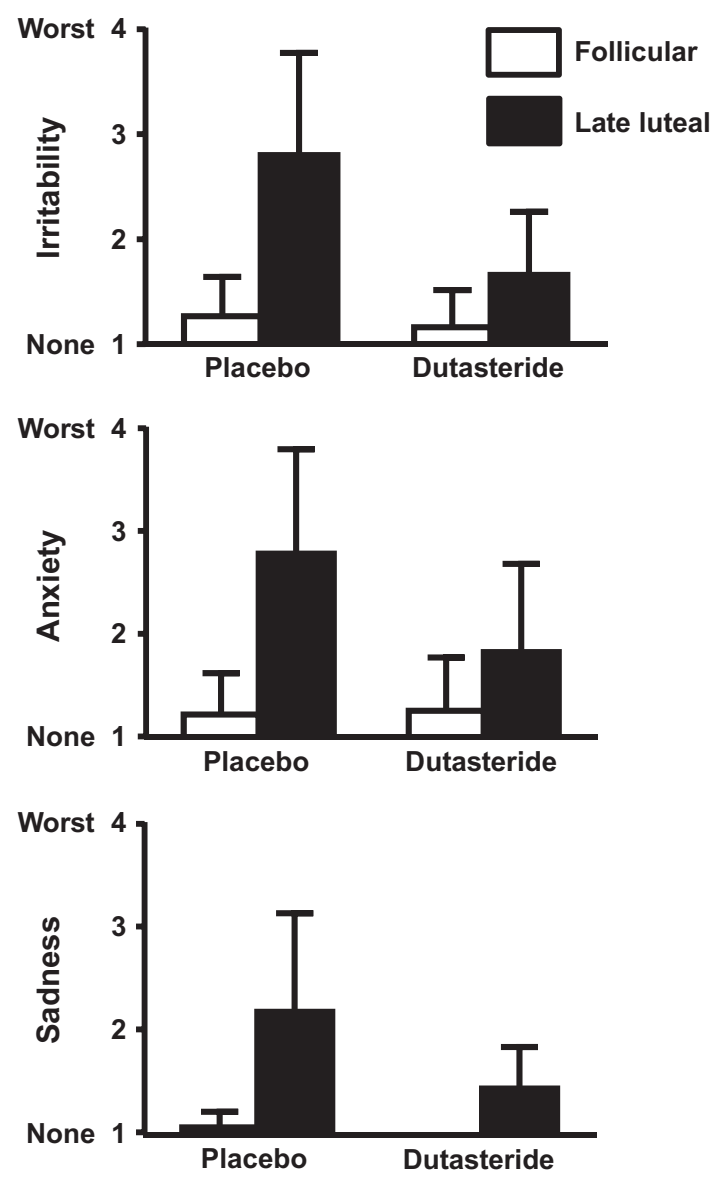

Figure 2 Weekly scores for the symptom of irritability, sadness and anxiety in women with premenstrual dysphoric disorder on high-dose dutasteride ( $2.5 \mathrm{mg}$ daily). Luteal symptom severity and menstrual cyclerelated symptom cyclicity were significantly reduced $(p<0.05)$ during highdose dutasteride compared with severity scores during placebo treatment. Significant reductions in the difference between week 4 (premenstrual, luteal) and week 2 (postmenstrual, follicular) symptom severity scores were observed in women with premenstrual dysphoric disorder (PMDD) during dutasteride compared with placebo treatments in daily rating form symptom scores of irritability $(p=0.04)$, anxiety $(p=0.03)$, and sadness $(p=0.04)$. The remission of PMDD symptoms and the reduction of symptom cyclicity occurred despite the absence of differences $(p>0.05)$ in either plasma progesterone levels or the number of anovulatory cycles between dutasteride and placebo conditions. 
PMDD during the menstrual cycle in which they received high-dose dutasteride. Finally, no significant differences in any symptom scores were identified in the control women between high-dose dutasteride and placebo treatments ( $p>0.05$ all comparisons).

Blood hormone levels on high-dose dutasteride. Plasma progesterone levels in women with PMDD and control women did not differ during dutasteride compared with placebo $(p=0.4)$, nor was there a significant difference in the number of cycles with progesterone levels $<2 \mathrm{ng} / \mathrm{ml}$ (Fisher exact $p=1.0$ ) (Table 3; Figure 3). Plasma progesterone levels were $<2 \mathrm{ng} / \mathrm{ml}$ in two women with PMDD during both dutasteride and placebo treatments, and in three control women during dutasteride $(n=3)$ and placebo $(n=1)$ (one control women had progesterone levels $<2$ in both treatment cycles). There were no significant differences in either follicular or luteal phase plasma estradiol levels between placebo and dutasteride treatments $(p=0.5)$.

There was a significant effect of treatment assignment in the difference between log-transformed follicular and luteal phase levels of allopregnanolone $(p=0.001)$ and $3 \alpha, 5 \alpha-$ androsterone $(p=0.03)$, reflecting an increase in the levels of these two neurosteroids from follicular to luteal phase during placebo but not during high-dose dutasteride. No differences in pregnenolone or the $5 \beta$-reduced steroids were observed across the luteal phase or following dutasteride treatment. Finally, during placebo treatment, plasma allopregnanolone levels (mean(SD)) in women with PMDD were 241.4 (209.6) $\mathrm{pg} / \mathrm{ml}$ and in controls $127.1(86.6) \mathrm{pg} / \mathrm{ml}$. These plasma levels need to be interpreted cautiously since the mean in PMDD reflects an outlier value (ie, high levels in one woman with PMDD) within a relatively small sample.

Tolerability and side effects. Dutasteride was well tolerated (Table 4), and neither sexual interest nor breast pain worsened during the one month exposure to dutasteride treatment. High-dose dutasteride had no significant effect on the numbers of days of menses. The mean (SD) days of menstrual flow at baseline and after one menstrual cycle of placebo and dutasteride were as follows: baseline (pre-study $=4.8(0.9)$ days; placebo $=5.0(1.6)$ days; dutasteride $=4.9(1.0)$ days. There was no significant difference in the duration of menses between placebo and dutasteride treatments (Student's $t$-test $=0.9, p=\mathrm{NS}$ ).

\section{DISCUSSION}

Several observations support the possible role of neurosteroids in the etiology of PMDD. First, studies report both abnormalities of peripheral neurosteroid levels (albeit inconsistently) and the neurosteroid response to stress in women with PMDD (Wang et al, 1996; Rapkin et al, 1997; Monteleone et al, 2000; Lombardi et al, 2004; Klatzkin et al, 2006). Second, women with PMDD show the following: lowered cortical GABA levels (Epperson et al, 2002); blunted luteal phase cortical inhibition (Smith et al, 2003); and altered sensitivities of saccadic eye velocity to both neurosteroids and benzodiazepines (Sundstrom et al, 1997a; Sundstrom et al, 1997b; Sundstrom et al, 1998). Finally, the most effective therapy for PMDD, selective serotonin reuptake inhibitors (SSRIs), activate $3 \alpha$-hydroxy steroid

Table 3 Plasma Hormone Levels in Women with Premenstrual Dysphoric Disorder and Control Women on Dutasteride (2.5 mg/day) and Placebo

Follicular and luteal phase plasma levels (arithmetic mean (SD))

Placebo

Follicular

Dutasteride

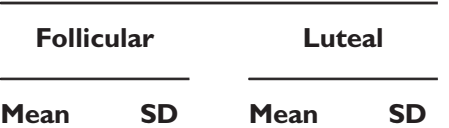

Follicular-luteal difference in plasma levels LSE (SE)

\begin{tabular}{llll}
\hline \multicolumn{2}{c}{ Placebo } & & \multicolumn{2}{c}{ Dutasteride } \\
\cline { 1 - 1 } & & & \\
\end{tabular}

Progesterone (ng/ml)

Pregnenolone (pg/ml)

Allopregnanolone (pg/ml)

Pregnanolone (pg/ml)

$3 \alpha, 5 \alpha$-Androsterone (pg/ml)

$3 \alpha, 5 \beta$-Androsterone $(\mathrm{pg} / \mathrm{ml})^{\mathrm{a}}$

Estradiol (pg/ml)

$\overline{\text { Mean }}$ SD $\overline{\text { Mean SD }}$

260.1
121.3
34.4
148.8
13.2
56.5

110.4
155.
27.3
88.5
7.5
34.

8.1
351.8

$$
189.5
$$

165.8

13.6

124.5

3.9
191.0
168.9
19.7
101.2
8.9
71.6

$\begin{array}{rr}264.9 & 89.6 \\ 140.2 & 148 . \\ 33.8 & 31.9 \\ 162.8 & 107.7 \\ 34.9 & 31.9 \\ 78.7 & 51.6\end{array}$

$\begin{array}{rrr} & 9.4 & 4.0 \\ 89.6 & 281.2 & 44.7 \\ 148.1 & 120.4 & 113.4 \\ 31.9 & 80.2 & 61.7 \\ 107.7 & 152.3 & 110.1 \\ 31.9 & 34.9 & 28.0 \\ 51.6 & 113.0 & 64.8\end{array}$

$\begin{array}{rr}4.0 & \\ 44.7 & 100.3 \\ 113.4 & 69.5 \\ 61.7 & 4.2 \\ 110.1 & 18.2 \\ 28.0 & 1.0 \\ 64.8 & 60.6\end{array}$

$\begin{array}{rrrc}100.3 & 45.0 & 24.9 & 45.0 \\ 69.5 & 26.1 & -18.5 & 26.1^{+} \\ 4.2 & 16.4 & 43.5 & 16.4 \\ 18.2 & 6.7 & -9.3 & 6.7^{\ddagger} \\ 1.0 & 6.1 & -1.8 & 5.4 \\ 60.6 & 26.7 & 26.9 & 26.7\end{array}$

There were no effects of diagnosis on plasma hormone levels, thus, values reported are those from both women with PMDD and control women. There was a significant trend effect of treatment assignment in the difference between follicular to luteal phase plasma levels of allopregnanolone $\left({ }^{+} p=0.05\right)$ which was significant when log-transformed values were analyzed $(p=0.00 \mathrm{I})$, and a significant effect of treatment in the difference between follicular to luteal phase plasma levels of $3 \alpha, 5 \alpha$ - androsterone $\left({ }^{\ddagger} p=0.03\right.$, (log-transformed values: $\left.p=0.10\right)$ ), reflecting an increase in the levels of these two neurosteroids from follicular to luteal phase during placebo but not during high-dose dutasteride. No significant differences in the follicular-luteal change of the raw or log-transformed plasma values of the $5 \beta$-metabolites, pregnenolone, or estradiol were observed between high-dose dutasteride and placebo. Plasma progesterone levels were not obtained during the follicular phase of the menstrual cycle. Progesterone values during the luteal phase did not differ between dutasteride and placebo $(p>0.05)$.

$a_{n}=$ six women, all other values $n=11$ (women with PMDD $(n=6)$ and control women $\left.(n=5)\right)$. 

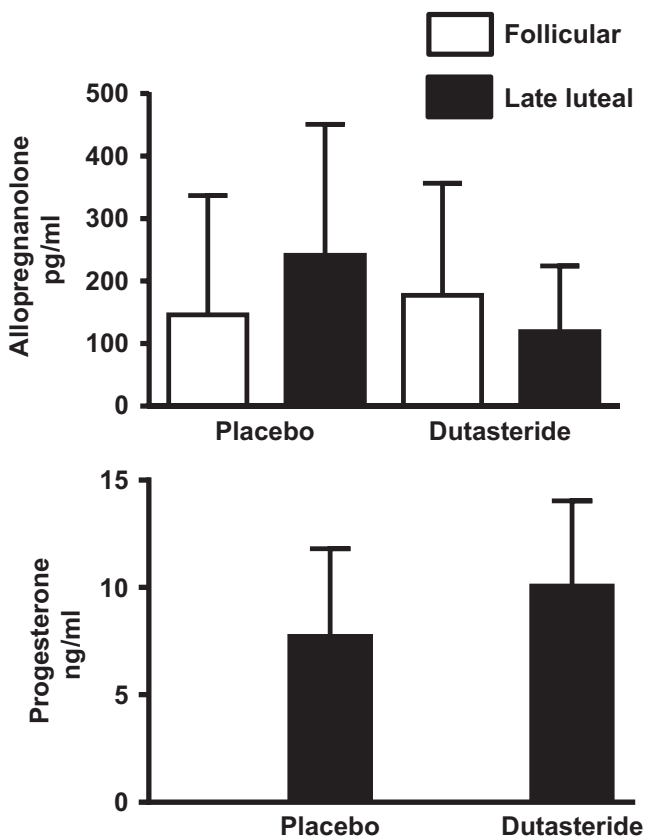

Figure 3 Plasma allopregnanolone and progesterone levels during dutasteride and placebo in women with premenstrual dysphoric disorder. Plasma progesterone levels in women with premenstrual dysphoric disorder (PMDD) did not differ during dutasteride compared with placebo $(p=0.4)$, nor was there a significant difference in the number of cycles with progesterone levels $<2 \mathrm{ng} / \mathrm{ml}$ (Fisher exact $p=1.0$ ). Plasma progesterone levels were $<2 \mathrm{ng} / \mathrm{ml}$ in two women with PMDD during both dutasteride and placebo treatments, and in three control women during dutasteride $(n=3)$ and placebo $(n=1$; one control women had progesterone levels $<2$ in both treatment cycles). There was a significant effect of treatment assignment in the difference between follicular to luteal phase levels of allopregnanolone $(p=0.05$ (raw values) and $p=0.00 \mathrm{I}$ (log transformed)) and $3 \alpha, 5 \alpha$ androsterone $(p=0.03)$, reflecting an increase in the levels of these two neurosteroids from the follicular to luteal phase during placebo but not during dutasteride. The percent changes (mean (SD)) in allopregnanolone levels from follicular to luteal phases were as follows: during placebo $170.9 \%$ (158.5) and during dutasteride (2.5 mg per day) $-12.8 \%$ (58.1). No differences between dutasteride and placebo were observed in the luteal increase in either the $5 \beta$-metabolites or pregnenolone. Despite the absence of any negative affective symptoms induced by dutasteride in the control women, the effects of dutasteride on the luteal phase increase in allopregnanolone were also observed in control women.

dehydrogenase-one of the synthetic enzymes involved in the conversion of progesterone to allopregnanolone (Griffin and Mellon, 1999), and SSRI administration is accompanied by increases in CSF allopregnanolone (Uzunova et al, 1998).

In this study, we observed that dutasteride at a daily dose of $2.5 \mathrm{mg}$, but not placebo, prevented the luteal phase increase in allopregnanolone and significantly reduced PMDD symptoms. The mitigation of PMDD symptoms occurred in the presence of ovulatory menstrual cycles, and there was no difference in plasma progesterone levels during the luteal phase of the placebo treatment compared with high-dose dutasteride. Neither effect was observed with the lower dose of dutasteride (ie, $0.5 \mathrm{mg}$ a day), nor were symptoms induced in control women.

High-dose dutasteride significantly decreased the core affective symptoms of irritability, anxiety, and sadness and some physical symptoms (bloating and food cravings). In addition to reducing luteal phase symptom severity in the women with PMDD, dutasteride also decreased the luteal to follicular symptom cyclicity, the hallmark of PMDD. Six of eight women no longer met criteria for PMDD during the menstrual cycle in which they received high-dose dutasteride. Dutasteride did not improve all symptoms examined (eg, mood swings and breast pain), which could suggest a limited target range for dutasteride in the treatment of PMDD. Alternatively, the one month cycle of treatment may have been insufficient for the full range of PMDD symptoms to respond to treatment. Dutasteride was well tolerated and neither sexual interest nor breast pain worsened during the one month exposure to dutasteride treatment. Indeed nonsignificant improvements were observed in these symptoms during dutasteride compared with placebo.

Consistent with its role as a $5 \alpha$-reductase inhibitor, highdose dutasteride blunted the expected luteal phase increase in plasma levels of allopregnanolone. Several other steroids are substrates for $5 \alpha$-reductase including testosterone, deoxycorticosterone, androstenedione, and 17 hydroxy-progesterone (Miller and Auchus, 2011). We cannot rule out alterations in these steroid metabolites as contributing to the therapeutic efficacy of dutasteride. For example, we observed that highdose dutasteride decreased levels of androsterone, a neuroactive steroid metabolite of the androgen androstenedione. Nonetheless, we can rule out a 'shunting' of metabolism to the $5 \beta$-pathway, as we found no increase in the $5 \beta$-neurosteroids following high-dose administration of dutasteride. Similarly, blocked metabolism of testosterone to DHT did not result in increased production of estradiol, the aromatized metabolite of testosterone.

In previous studies of the role of ovarian steroids in PMDD, we reported that changes in ovarian steroid concentrations within the normal range directly trigger affective symptoms, but only in a subset of women with histories of PMDD (Schmidt et al, 1998). In this study, our findings suggest that the luteal phase increase in allopregnanolone mediates the change in negative affective state in women with PMDD during the luteal phase. However the same effects on behavior were not present in asymptomatic controls with no past history of PMDD who underwent the identical protocol. These data suggest that women with PMDD have a differential sensitivity to changes in allopregnanolone levels, perhaps at the level of the GABA $\mathrm{R}$ (Backström et al, 2011) (possibly reflecting an increased expression of the $\alpha 4 \beta \delta$ GABA-A receptor in PMDD that could result in allopregnanolonemediated decreased inhibition and anxiety-like behaviors (Shen et al, 2007)), or secondary to an altered metabolism of allopregnanolone and other neurosteroids within the CNS. The role of progesterone and its $5 \alpha$-reduced metabolites in affective regulation is supported by several findings in humans (Schiller et al, 2014). First, progesterone and allopregnanolone are differentially localized in human brain with high concentrations reported in several brain regions involved in affective regulation including the amygdala and hypothalamus (Bixo et al, 1997). Second, the mRNA expression profiles and activities (in vitro) of both $5 \alpha$-reductase (type 1) and $3 \alpha$ hydroxysteroid dehydrogenase (critical in the formation of allopregnanolone) have been identified in human temporal cortex, amygdala, and hippocampus (Stoffel-Wagner et al, 2000; Stoffel-Wagner, 2001; Steckelbroeck et al, 2001; Pelletier, 
Table 4 Tolerability and Side Effects After One Menstrual Cycle of Placebo and High Dose (HD) Dutasteride (2.5 mg/day). Numbers of Women Reporting at Least Moderate Symptoms (Scores of 2 or Greater)*

\begin{tabular}{|c|c|c|c|c|c|}
\hline \multirow[t]{2}{*}{ Symptom on HD } & \multicolumn{2}{|c|}{ Control $(n=8)$} & \multirow[t]{2}{*}{ Symptom on HD } & \multicolumn{2}{|c|}{ PMDD $(n=8)$} \\
\hline & Placebo & Dutasteride & & Placebo & Dutasteride \\
\hline Poor appetite & 0 & 0 & Poor appetite & 0 & 0 \\
\hline Dry mouth & 0 & 0 & Dry mouth & 0 & 0 \\
\hline Tremor & 0 & 0 & Tremor & 0 & 0 \\
\hline Weakness & 0 & 0 & Weakness & 0 & 0 \\
\hline Fatigue & 0 & 0 & Fatigue & । & 2 \\
\hline Headache & 0 & I & Headache & I & 2 \\
\hline Itching & 0 & 0 & Itching & 0 & 0 \\
\hline Stomachache & 0 & 0 & Stomachache & 0 & I \\
\hline Increased urinary frequency & 0 & I & Increased urinary frequency & 0 & 0 \\
\hline Heart skipping beats & 0 & 0 & Heart skipping beats & 0 & I \\
\hline Racing heart & 0 & 0 & Racing heart & 0 & I \\
\hline Dizziness & 0 & I & Dizziness & I & 0 \\
\hline Blurry vision & 0 & 0 & Blurry vision & 0 & 0 \\
\hline Decreased sex drive & 0 & 0 & Decreased sex drive & । & I \\
\hline Increased sex drive & 0 & 0 & Increased sex drive & 0 & 1 \\
\hline Increased sweating & 0 & 0 & Increased sweating & 0 & 0 \\
\hline Rash & 0 & 0 & Rash & 0 & 0 \\
\hline Breast tenderness & 0 & 0 & Breast tenderness & 0 & 0 \\
\hline
\end{tabular}

Dutasteride was well tolerated and neither sexual interest nor breast pain worsened during the one month exposure to dutasteride treatment. *Scores in Table 4 represent the numbers of women who reported symptom severity scores $>2$ (range of scale: $0=$ none to $3=$ much).

2010). Third, during an emotional appraisal task in healthy men treated with pregnenolone, increased allopregnanolone plasma levels were accompanied by altered connectivity between the medial PFC and the amygdala, components of a circuit implicated as dysfunctional in depression (Sripada et al, 2013). Fourth, postmortem studies observed decreased $5 \alpha$-reductase mRNA expression in the PFC of depressed men and women compared with non-depressed controls (Agis-Balboa et al, 2014). Thus, the human brain may selectively concentrate and synthesize neurosteroids to regulate both reproductive and affective behaviors. Finally, variation in the gene for $5 \alpha$-reductase type 1 within the hair follicles of women with polycystic ovarian syndrome has been associated with the severity of hirsutism (Goodarzi et al, 2006). Thus, the altered phenotypic response to changes in allopregnanolone observed in women with PMDD could represent altered levels of allopregnanolone consequent to tissue specific polymorphic variants in the gene for type $15 \alpha$-reductase.

Dutasteride has been reported to have therapeutic benefits in a range of neuropsychiatric illnesses, and some of these effects are thought to be independent of its actions on androgen metabolism (Gulinello and Smith, 2003; Bortolato et al, 2008; Paba et al, 2011; Devoto et al, 2012). Thus, although no study has directly evaluated the CNS penetrance of dutasteride, both clinical reports and studies in rodents (GlaxoSmithKline Research Triangle Park NC, 2005) suggest that dutasteride does cross the blood-brain barrier.

This study was designed to test a specific hypothesis and not evaluate the efficacy of a new treatment for PMDD. Nonetheless, it is conceivable that dutasteride might be an alternative treatment in women with PMDD, but there are obviously additional considerations and risks. The primary 
risk is that of risk to the fetus if a woman becomes pregnant, the normal process of masculinization of the fetus by DHT would be prevented, and several developmental abnormalities would be predicted to occur including hypospadias. Thus women of child-bearing age should not risk becoming pregnant while taking dutasteride and for at least 6 months after stopping dutasteride. That said, dutasteride appears to be well tolerated in men, with the primary adverse effects reported are those related to sexual function (Olsen et al, 2006; GlaxoSmithKline Research Triangle Park NC, 2014). The potential therapeutic benefits of dutasteride warrant further investigation in a larger randomized, controlled trial. Indeed, dutasteride could be an effective treatment option for the $30-60 \%$ of women with PMDD who either do not respond to traditional SSRI therapy or develop treatment limiting side effects to SSRI.

In conclusion, our preliminary findings suggest both a novel therapeutic intervention for women with PMDD and a potential mechanism involving changes in neurosteroid levels in the pathogenesis of the PMDD.

\section{FUNDING AND DISCLOSURE}

The Intramural Research Program of the NIMH, NIH supported this study and GlaxoSmithKline Pharmaceuticals provided material support; however, neither institution was involved in the design and conduct of the study; collection, management, analysis, and interpretation of the data; and preparation, review, or approval of the manuscript. Dr Nieman receives compensation from UpToDate, Inc. for contributing to the adrenal section. Dr Schmidt had full access to all the data in this study and takes responsibility for the integrity of the data and the accuracy of the data analysis. Drs. Martinez, Schmidt and Koziol performed data analyses for this study.This work was written as part of Peter J Schmidt's official duties as a Government employee. The views expressed in this article do not necessarily represent the views of the NIMH, NIH, HHS, or the United States Government.This research was supported by the Intramural Research Program of the NIMH, NIH. The authors declare no conflict of interest.

\section{ACKNOWLEDGMENTS}

We wish to acknowledge Ms Linda Schenkel for data management support, and GlaxoSmithKline Pharmaceuticals, Research Triangle Park, NC for supplies of dutasteride for this study.

\section{REFERENCES}

Agis-Balboa RC, Guidotti A, Pinna G (2014). 5 $\alpha$-reductase type I expression is downregulated in the prefrontal cortex/Brodmann's area 9 (BA9) of depressed patients. Psychopharmacology 231: 3569-3580.

American Psychiatric Association (1994). Diagnostic and Statistical Manual of Mental Disorders, Fourth Edition, American Psychiatric Association: Washington, DC, USA.

American Psychiatric Association (2013). Diagnostic and Statistical Manual of Mental Disorders, Fifth Edition, American Psychiatric Association: Arlington, VA, USA.
Backström T, Haage D, Löfgren M, Johansson IM, Strömberg J, Nyberg S et al (2011). Paradoxical effects of GABA-A modulators may explain sex steroid induced negative mood symptoms in some persons. Neuroscience 191: 46-54.

Bixo M, Andersson A, Winblad B, Purdy RH, Backström T (1997). Progesterone, 5 $\alpha$-pregnane-3,20-dione and $3 \alpha$-hydroxy-5 $\alpha$-pregnane-20-one in specific regions of the human female brain in different endocrine states. Brain Res 764: 173-178.

Bortolato M, Frau R, Orru M, Bourov Y, Marrosu F, Mereu G et al (2008). Antipsychotic-like properties of 5 - $\alpha$-reductase inhibitors. Neuropsychopharmacology 33: 3146-3156.

Bramson HN, Hermann D, Batchelor KW, Lee FW, James MK, Frye SV (1997). Unique preclinical characteristics of GG745, a potent dual inhibitor of 5AR. J Pharmacol Exp Ther 282: 1496-1502.

Devoto P, Frau R, Bini V, Pillolla G, Saba P, Flore G et al (2012). Inhibition of $5 \alpha$-reductase in the nucleus accumbens counters sensorimotor gating deficits induced by dopaminergic activation. Psychoneuroendocrinology 37: 1630-1645.

Endicott J, Nee J, Cohen J, Halbreich U (1986). Premenstrual changes: patterns and correlates of daily ratings. J Affect Disord 10: 127-135.

Epperson CN, Haga K, Mason GF, Sellers E, Gueorguieva R, Zhang $\mathrm{W}$ et al (2002). Cortical $\gamma$-aminobutyric acid levels across the menstrual cycle in healthy women and those with premenstrual dysphoric disorder: a proton magnetic resonance spectroscopy study. Arch Gen Psychiatry 59: 851-858.

First MB, Spitzer RL, Gibbon M, Williams JBW (2002). Structured Clinical Interview for DSM-IV-TR Axis I Disorders, Research Version, Patient Edition (SCID-I/P). Biometric Research, New York State, Psychiatric Institute: New York.

GlaxoSmithKline Research Triangle Park NC (2014). Avodart prescribing information. https://www.gsksource.com/pharma/ content/dam/GlaxoSmithKline/US/en/Prescribing_Information/ Avodart/pdf/AVODART-PI-PIL.PDF.

GlaxoSmithKline Research Triangle Park NC (2005). GI198745: Quantitative whole-body autoradiography following single and repeated administration to the mouse.

Goodarzi MO, Shah NA, Antoine HJ, Pall M, Guo X, Azziz R (2006). Variants in the $5 \alpha$-reductase type 1 and type 2 genes are associated with polycystic ovary syndrome and the severity of hirsutism in affected women. J Clin Endocrinol Metab 91: 4085-4091.

Griffin LD, Mellon SH (1999). Selective serotonin reuptake inhibitors directly alter activity of neurosteroidogenic enzymes. Proc Natl Acad Sci USA 96: 13512-13517.

Gulinello M, Gong QH, Li X, Smith SS (2001). Short-term exposure to a neuroactive steroid increases $\alpha 4$ GABAA receptor subunit levels in association with increased anxiety in the female rat. Brain Res 910: 55-66.

Gulinello M, Smith SS (2003). Anxiogenic effects of neurosteroid exposure: sex differences and altered GABAA receptor pharmacology in adult rats. J Pharmacol Exp Ther 305: 541-548.

Hilsenroth MJ, Ackerman SJ, Blagys MD, Baumann BD, Baity MR, Smith SR et al (2000). Reliability and validity of DSM-IV axis V. Am J Psychiatry 157: 1858-1863.

Klatzkin RR, Morrow AL, Light KC, Pedersen CA, Girdler SS (2006). Histories of depression, allopregnanolone responses to stress, and premenstrual symptoms in women. Biol Psychol 71: 2-11.

Lombardi I, Luisi S, Quirici B, Monteleone P, Bernardi F, Liut M et al (2004). Adrenal response to adrenocorticotropic hormone stimulation in patients with premenstrual syndrome. Gynecol Endocrinol 18: 79-87.

Maguire JL, Stell BM, Rafizadeh M, Mody I (2005). Ovarian cyclelinked changes in GABAA receptors mediating tonic inhibition alter seizure susceptibility and anxiety. Nat Neurosci 8: 797-804.

Majewska MD, Harrison NL, Schwartz RD, Barker JL, Paul SM (1986). Steroid hormone metabolites are barbiturate-like modulators of the GABA receptor. Science 232: 1004-1007. 
Miller WL, Auchus RJ (2011). The molecular biology, biochemistry, and physiology of human steroidogenesis and its disorders. Endocr Rev 32: 81-151.

Monteleone P, Luisi S, Tonetti A, Bernardi F, Genazzani AD, Luisi $\mathrm{M}$ et al (2000). Allopregnanolone concentrations and premenstrual syndrome. Eur J Endocrinol 142: 269-273.

Olsen EA, Hordinsky M, Whiting D, Stough D, Hobbs S, Ellis ML et al (2006). The importance of dual $5 \alpha$-reductase inhibition in the treatment of male pattern hair loss: results of a randomized placebo-controlled study of dutasteride versus finasteride. $\mathrm{J} \mathrm{Am}$ Acad Dermatol 55: 1014-1023.

Paba S, Frau R, Godar SC, Devoto P, Marrosu F, Bortolato M (2011). Steroid $5 \alpha$-reductase as a novel therapeutic target for schizophrenia and other neuropsychiatric disorders. Curr Pharm Des 17: 151-167.

Paul SM, Purdy RH (1992). Neuroactive steroids. FASEB J 6: 2311-2322.

Pelletier G (2010). Steroidogenic enzymes in the brain: morphological aspects. Prog Brain Res 181: 193-207.

Porcu P, O'Buckley TK, Alward SE, Marx CE, Shampine LJ, Girdler SS et al (2009). Simultaneous quantification of GABAergic $3 \alpha, 5 \alpha /$ $3 \alpha, 5 \beta$ neuroactive steroids in human and rat serum. Steroids 74 : 463-473.

Rapkin AJ, Morgan M, Goldman L, Brann DW, Simone D, Mahesh VB (1997). Progesterone metabolite allopregnanolone in women with premenstrual syndrome. Obstet Gynecol 90: 709-714.

Rubinow DR, Roy-Byrne PP, Hoban MC, Gold PW, Post RM (1984). Prospective assessment of menstrually related mood disorders. Am J Psychiatry 141: 684-686.

Rubinow DR, Schmidt PJ (2006). Gonadal steroid regulation of mood: the lessons of premenstrual syndrome. Front Neuroendocrinol 27: 210-216.

Schiller CE, Schmidt PJ, Rubinow DR (2014). Allopregnanolone as a mediator of affective switching in reproductive mood disorders. Psychopharmacology 231: 3557-3567.

Schmidt PJ, Nieman LK, Danaceau MA, Adams LF, Rubinow DR (1998). Differential behavioral effects of gonadal steroids in women with and in those without premenstrual syndrome. N Engl J Med 338: 209-216.

Schmidt PJ, Purdy RH, Moore PH Jr., Paul SM, Rubinow DR (1994). Circulating levels of anxiolytic steroids in the luteal phase in women with premenstrual syndrome and in control subjects. J Clin Endocrinol Metab 79: 1256-1260.

Shen H, Gong QH, Aoki C, Yuan M, Ruderman Y, Dattilo M et al (2007). Reversal of neurosteroid effects at $\alpha 4 \beta 2 \delta$
GABAA receptors triggers anxiety at puberty. Nat Neurosci 10: 469-477.

Shen H, Gong QH, Yuan M, Smith SS (2005). Short-term steroid treatment increases delta GABAA receptor subunit expression in rat CA1 hippocampus: pharmacological and behavioral effects. Neuropharmacology 49: 573-586.

Smith MJ, Adams LF, Schmidt PJ, Rubinow DR, Wassermann EM (2003). Abnormal luteal phase excitability of the motor cortex in women with premenstrual syndrome. Biol Psychiatry 54: 757-762.

Sripada RK, Marx CE, King AP, Rampton JC, Ho SS, Liberzon I (2013). Allopregnanolone elevations following pregnenolone administration are associated with enhanced activation of emotion regulation neurocircuits. Biol Psychiatry 73: 1045-1053.

Steckelbroeck S, Watzka M, Reichelt R, Hans VHJ, StoffelWagner B, Heidrich DD et al (2001). Characterization of the $5 \alpha$-reductase- $3 \alpha$-hydroxysteroid dehydrogenase complex in the human brain. J Clin Endocrinol Metab 86: 1324-1331.

Stoffel-Wagner B (2001). Neurosteroid metabolism in the human brain. Eur J Endocrinol 145: 669-679.

Stoffel-Wagner B, Beyenburg S, Watzka M, Blümcke I, Bauer J, Schramm J et al (2000). Expression of 5 $\alpha$-reductase and $3 \alpha$-hydroxisteroid oxidoreductase in the hippocampus of patients with chronic temporal lobe epilepsy. Epilepsia 41: 140-147.

Sundstrom I, Andersson A, Nyberg S, Ashbrook D, Purdy RH, Backstrom T (1998). Patients with premenstrual syndrome have a different sensitivity to a neuroactive steroid during the menstrual cycle compared to control subjects. Neuroendocrinology 67: 126-138.

Sundstrom I, Ashbrook D, Backstrom T (1997a). Reduced benzodiazepine sensitivity in patients with premenstrual syndrome: a pilot study. Psychoneuroendocrinology 22: 25-38.

Sundstrom I, Nyberg S, Backstrom T (1997b). Patients with premenstrual syndrome have reduced sensitivity to midazolam compared to control subjects. Neuropsychopharmacology 17: $370-381$.

Uzunova V, Sheline Y, Davis JM, Rasmusson A, Uzunov DP, Costa $\mathrm{E}$ et al (1998). Increase in the cerebrospinal fluid content of neurosteroids in patients with unipolar major depression who are receiving fluoxetine or fluvoxamine. Proc Natl Acad Sci USA 95: 3239-3244.

Wang M, Seippel L, Purdy RH, Bäckström T (1996). Relationship between symptom severity and steroid variation in women with premenstrual syndrome: study on serum pregnenolone, pregnenolone sulfate, $5 \alpha$-pregnane-3,20-dione and $3 \alpha$-hydroxy5 $\alpha$-pregnan-20-one. J Clin Endocrinol Metab 81: 1076-1082. 